\title{
2-甲酰噻吩缩氨基硫腿及其芳基钉配合物的合成、结构与抗癌活性研究
}

\author{
刘利锋 ${ }^{a}$ 李培源 $*, b$ 钱全全 ${ }^{a}$ 雷晓琳 ${ }^{a}$ 黄钰湘 ${ }^{a}$ \\ 周 泉 ${ }^{a}$ 黄 珊 ${ }^{a}$ 肖 琦 ${ }^{a}$ 苏 炜*,
}

( ${ }^{a}$ 北部湾环境演变与资源利用教育部重点实验室 广西师范学院化学与生命科学学院 南宁 530001)

( $b$ 广西中医药大学药学院 南宁 530001)

\begin{abstract}
摘要 合成了 2-甲酰噻吩缩氨基硫腿化合物 $\mathbf{L 1}, \mathbf{L 2}$ 和它们的芳基钓配合物 $\left[\left(\eta^{6}-p\right.\right.$-cymene) Ru $\left.{ }^{\mathrm{II}}(\mathrm{TSC}) \mathrm{Cl}\right] \mathrm{Cl}$ (1: TSC $=\mathbf{L 1}$, 2: $\mathrm{TSC}=\mathbf{L 2} 2$ ), 其结构经 ${ }^{1} \mathrm{H} N \mathrm{NR}, \mathrm{MS}$ 和元素分析确认. 通过单晶培养得到了 $\mathbf{1}$ 的晶体, 并测定其晶体结构. 凝胶电泳实 验表明, L1 和 L2 51 和 2 对 $\mathrm{pBR} 322$ 质粒 DNA 存在不同的作用机理. 采用 MTT 法测定了这些化合物对 3 种人体肿瘤 细胞株(SGC7901, BEL7404 和 CNE-1)的细胞毒活性. 其中化合物 1 对人鼻咽癌细胞株(CNE-1)的有较好的抑制活性, 其 $\mathrm{IC}_{50}$ 值为 $27.8 \mu \mathrm{mol} \cdot \mathrm{L}^{-1}$.
\end{abstract}

关键词 芳基钓配合物; 氨基硫嫝; 抗肿瘤

\section{Synthesis, Structure, Anticancer Activity of 2-Formylthiophene Thiosemicarbazones and Their Organometallic Ruthenium Complexes}

\author{
Liu, Lifeng ${ }^{a}$ \\ Li, Peiyuan*,b \\ Qian, Quanquan ${ }^{a}$ \\ Lei, Xiaolin ${ }^{a}$ \\ Zhou, Quan ${ }^{a}$ \\ Huang, Shan ${ }^{a}$ \\ Xiao, $\mathrm{Qi}^{a}$ \\ $\mathrm{Su}, \mathrm{Wei}^{*, a}$ \\ ( ${ }^{a}$ Key Laboratory of Beihu Gulf Environment Change and Resource Utilization, Ministry of Education; \\ College of Chemistry and Life Science, Guangxi Teachers Education University, Nanning 530001) \\ ( ${ }^{b}$ College of Pharmacy, Guangxi University of Chinese Medicine, Nanning 530001)
}

Huang, Yuxiang ${ }^{a}$

\begin{abstract}
Two 2-formylthiophene thiosemicarbazone compounds (L1, L2) and the ruthenium(II) arene complexes $\left[\left(\eta^{6}-p\right.\right.$-cymene $\left.) \mathrm{Ru}^{\mathrm{II}}(\mathrm{TSC}) \mathrm{Cl}\right] \mathrm{Cl}(\mathbf{1}: \mathrm{TSC}=\mathbf{L 1}, \mathbf{2}: \mathrm{TSC}=\mathbf{L 2})$ have been synthesized. The complexes were characterized by ${ }^{1} \mathrm{H}$ NMR, MS and elemental analysis. The crystal structure of $\mathbf{1}$ is determined by single-crystal X-ray diffraction. The gel electrophoresis results show that the reaction mechanism for $\mathbf{L 1} / \mathbf{L} 2$ and $\mathbf{1} / \mathbf{2}$ with pBR322 plasmid DNA is different. The in vitro anticancer activities of these complexes have been evaluated against three human cancer cell lines (SGC7901, BEL7404 and $\mathrm{CNE}-1$ ), and $\mathbf{1}$ is proved to be the most efficient inhibitor with $\mathrm{IC}_{50}$ values of $27.3 \mu \mathrm{mol} \cdot \mathrm{L}^{-1}$ against CNE-1.
\end{abstract}

Keywords Ru-arene complex; thiosemicarbazone; antitumor

氨基硫腿(TSC)及其金属配合物具有良好的生物活 性, 如抗菌、抗病毒、抗真菌和抗肿瘤活性等, 从而受 到人们的广泛关注 ${ }^{[1]}$. 近年来, 科研工作者合成出大量 的 TSC 衍生物并对其抗肿瘤活性进行了评估 ${ }^{[2]}$. 研究发 现, TSC 与铜、铁等过渡金属配位形成配合物后通常表
现出比单独的 TSC 配体更强的生物活性 ${ }^{[3]}$, 这是由于与 金属离子的配位后 TSC 化合物的生物活性得以进一步 修正和增强 ${ }^{[4]}$.

作为一类低毒高效的金属基抗肿瘤化合物，钉配合 物被认为是具有广阔应用前景的抗癌药物而成为研究

* E-mail: aaasuwei@yahoo.com.cn; lipearpear@yahoo.cn

Received January 12, 2013; revised February 20, 2013; published online February 22, 2013.

Project supported by the National Natural Science Foundation of China (Nos. 20961001, 21261005, 21203035, 51263002), the Key Project of Chinese Ministry of Education (No. 2010168), the Natural Science Foundation of Guangxi Province (Nos. 2010GXNSFB013014, 2012GXNSFAA053194), the Scientific Research Fund of Guangxi Traditional Chinese Medical University (No. 2012024) and the Key Laboratory of Beibu Gulf Environment Change and Resources Utilization (Guangxi Teachers Education University), Ministry of Education, China.

国家自然科学基金(Nos. 20961001，21261005，21203035，51263002)、教育部科学技术研究重点(No. 2010168)、广西自然科学基金(Nos. 2010GXNSFB013014, 2012GXNSFAA053194)、广西中医药大学科研基金(No. 2012024)和北部湾环境演变与资源利用教育部重点实验室资助项目. 
的热点 ${ }^{[5]}$. [HIm] $]$ trans $-\mathrm{RuCl}_{4}(\mathrm{DMSO})$ (Im)] (NAMI-A)和 $[\mathrm{ImH}]\left[\right.$ trans $\left.-\mathrm{RuCl}_{4}(\mathrm{Im})_{2}\right](\mathrm{KP} 1019)$ 这两个钓配合物成功 进入抗肿瘤临床试验 ${ }^{[6]}$ 更是大大激发了人们的研究热 情. 近年来, 芳基钓有机金属化合物因其优良的抗肿瘤 活性而受到广泛关注 ${ }^{[7]}$. 该类化合物 $\left[\left(\eta^{6}\right.\right.$-arene $) \mathrm{Ru}^{\mathrm{II}}(\mathrm{X})-$ $(\mathrm{Y})(\mathrm{Z})]^{+}$为半三明治型的结构, arene 为六元芳基环, $\mathrm{X}, \mathrm{Y}$ 和 $\mathrm{Z}$ 为另外三个配位点. 乙二胺、乙酰丙酮、联吡啶、 喹诺酮等不同配体与芳基钉片断配位后的细胞毒性研 究表明, 配体的本征性质对抗肿瘤活性有着非常重要的 影响 ${ }^{[8]}$. 大部分的研究观点认为, 此类芳基钓化合物其 中一个单齿配体 (一般为 $\mathrm{Cl}$ 离子) 解离后, 与 $\mathrm{DNA}$ 螺旋 的鸟嘌呤上的 N(7)配位, 由此破坏了 DNA 的结构, 干 扰 DNA 的转录和复制 ${ }^{[9]}$. 另外, 芳基钓化合物对 DNA 螺旋的嵌入机制也对分子的抗肿瘤作用起到非常重要 的作用 ${ }^{[10]}$. 还有一些研究发现, 芳基钓化合物在生理条 件下强烈的催化氧化作用也可能是这种化合物抗癌活 性的重要机制 ${ }^{[11]}$. 这些重要的研究结果, 为芳基钉类抗 肿瘤药物的设计与合成研究提供了非常有益的参考. 与 此同时, 含 TSCs 配体的芳基钓配合物也被合成用以进 行抗肿瘤活性研究 ${ }^{[4 a, 12]}$. 其中, Beckford 等 ${ }^{[12 a]}$ 还进一步 研究了该类化合物 $\left\{\left[\left(\eta^{6}-p\right.\right.\right.$-cymene $) \mathrm{Ru}($ piperonal thiosemicarbazones) $\mathrm{Cl}] \mathrm{Cl}\}$ 与小牛胸腺 DNA、人血清白蛋白 (HSA)和 pBR322 质粒 DNA 的相互作用以及对拓扑异构 酶 II 的抑制作用. 这些研究表明, TSC 芳基钓配合物是 一类具有潜在应用前景的抗肿瘤药物.

在本工作中, 我们合成两个 2-甲酰噻吩缩氨基硫艮 的芳基钓配合物 1, 2 (Eq. 1), 研究了配合物 1 的单晶结 构, 并对照研究了这些化合物与它们的 2-甲酰噻吩缩氨 基硫嫝配体 $\mathbf{L 1}, \mathbf{L 2}^{[11]}$ 对 DNA 的切割作用及其体外抗肿 瘤活性.
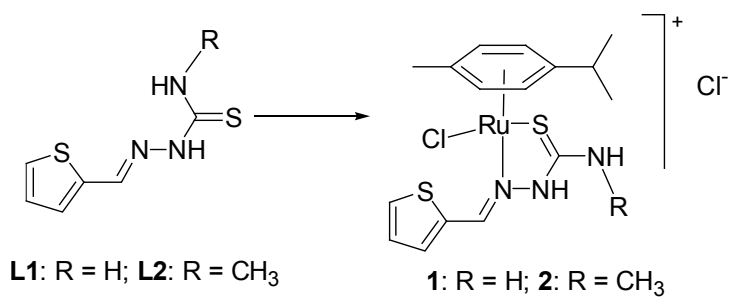

\section{1 结果与讨论}

\section{1 合成与表征}

2-甲酰噻吩缩氨基硫䐂化合物 L1, L2 与它们的芳 基钉配合物 $\mathbf{1}, \mathbf{2}$ 的合成都是根据文献[12，13]报道的反 应进行. 2-甲酰噻吩缩氨基硫脲是通过相应氨基硫脲与 2-甲酰噻吩缩合而成; 然后进一步与 [ $\eta^{6}-p$-cymene)$\left.\mathrm{RuCl}_{2}\right]_{2}$ 反应得到相应的芳基钓配合物 $\mathbf{1}, \mathbf{2}$. 所得到的新 化合物都经过核磁共振 $\left({ }^{1} \mathrm{H}\right.$ NMR)、紫外(UV-vis)、质谱
(ESI-MS)和元素分析的表征确认.

\section{2 晶体结构}

我们通过石油醚扩散法得到了芳基钓配合物 1 的单 晶. 晶体结构数据存于英国剑桥数据中心, CCDC 号为 993970. 单晶数据及结构精修参数见表 1 .

表 1 配合物的 1 晶体结构数据及结构精修参数

Table 1 Crystal data and stucture refinement for 1

\begin{tabular}{|c|c|}
\hline Complex & 1 \\
\hline CCDC No. & 993970 \\
\hline Formula & $\mathrm{C}_{16} \mathrm{H}_{21} \mathrm{~N}_{3} \mathrm{Cl}_{2} \mathrm{RuS}_{2}$ \\
\hline Temperature/K & $293(2)$ \\
\hline$M_{\mathrm{r}}$ & 491.46 \\
\hline Crystal system & Monoclinic \\
\hline Space group & $C 2 / c$ \\
\hline$a / \AA$ & $16.8389(9)$ \\
\hline$b / \AA$ & $8.2803(3)$ \\
\hline$c / \AA ̊$ & $28.8031(9)$ \\
\hline$\beta /\left(^{\circ}\right)$ & $95.115(4)$ \\
\hline$V / \AA^{3}$ & $4000.1(3)$ \\
\hline$Z$ & 8 \\
\hline$D_{\text {calcd }}\left(\mathrm{Mg} \mathrm{m}^{-3}\right)$ & 1.562 \\
\hline Crystal size $/ \mathrm{mm}^{3}$ & $0.41 \times 0.23 \times 0.12$ \\
\hline$F(000)$ & 1817 \\
\hline$\mu / \mathrm{mm}^{-1}$ & 1.261 \\
\hline$R_{\mathrm{int}}$ & 0.0417 \\
\hline Data/restraints/parameters & $4092 / 0 / 220$ \\
\hline$h$ range & $-20 \leqslant h \leqslant 20$ \\
\hline$k$ range & $-10 \leqslant k \leqslant 10$ \\
\hline$l$ range & $-36 \leqslant l \leqslant 33$ \\
\hline$\theta$ range $/\left(^{\circ}\right)$ & 5.62 to 52.74 \\
\hline Reflns collected & 16027 \\
\hline Reflns independent & 4092 \\
\hline GOF (S) & 1.081 \\
\hline$R_{1} / w R_{2}[I \geqslant 2 \sigma(I)]$ & $0.0378 / 0.0833$ \\
\hline$R_{1} / w R_{2}$ [all data] & $0.0510 / 0.0894$ \\
\hline
\end{tabular}

分子结构图见图 1. 部分键长与键角数据列于表 2 . 1 的晶体属于单斜晶系的 $C 2 / c$ 空间群. 金属钓离子(II) 同时与一个芳烃配体(甲基异丙基苯)、一个氯离子和一 个 $\mathrm{N}, \mathrm{S}$ 双齿配体配位，形成类似 “三脚琴登” 的几何构 型的一价阳离子. 钉原子与 “登面” 芳基环中心的距离 为 $1.696 \AA$, 三个 “登腿” 的长度分别为: $\mathrm{Ru}(1)-\mathrm{Cl}(1)$ $2.414 \AA ; \mathrm{Ru}(1)-\mathrm{S}(1) 2.363 \AA$ 和 $\mathrm{Ru}(1)-\mathrm{N}(3) 2.125 \AA$, 与已报道的 TSC 芳基钉配合物相似. TSC 配体上的 $\mathrm{C}(1)-\mathrm{S}(1)$ 键键长为 $1.694 \AA$, 属于典型的双键键长. 与 其相应 TSC 单体 $\mathbf{L 1}^{[13 a]}$ 相比, $\mathbf{1}$ 的 TSC 配体显示出不同 的构象, 它的 $\mathrm{S}(1)$ 与 $\mathrm{N}(3)$ 在配体主干的同一侧. 这种构 象的改变更有利于 $\mathrm{S}(1)$ 与 $\mathrm{N}(3)$ 这两个原子与金属的配 位. 由单个游离的氯离子构成的阴离子与 TSC 配体上 的两个 $\mathrm{N}$ 上的氢原子形成氢键: $\mathrm{N}(1)-\mathrm{H}(1 \mathrm{a}) \cdots \mathrm{Cl}(2)$ $(2.511 \AA)$ 和 $\mathrm{N}(2)-\mathrm{H} \cdots \mathrm{Cl}(2)(2.344 \AA)$. 


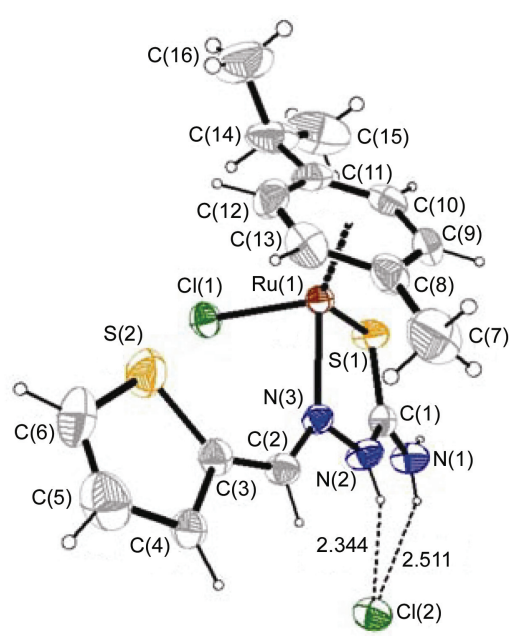

图 1 化合物 1 的晶体结构图

Figure 1 Crystal structure of 1

表 21 的部分键长 $(\AA)$ 和键角 $\left(^{\circ}\right)$

Table 2 Selected bond lengths $(\AA)$ and angles $\left({ }^{\circ}\right)$ in $\mathbf{1}$

\begin{tabular}{lc}
\hline $\mathrm{Ru}(1)-$ centroid & $1.6961(3)$ \\
$\mathrm{Ru}(1)-\mathrm{S}(1)$ & $2.3627(12)$ \\
$\mathrm{Ru}(1)-\mathrm{Cl}(1)$ & $2.4136(10)$ \\
$\mathrm{Ru}(1)-\mathrm{N}(3)$ & $2.1252(26)$ \\
$\mathrm{N}(3)-\mathrm{Ru}(1)-\mathrm{S}(1)$ & $81.856(82)$ \\
$\mathrm{S}(1)-\mathrm{Ru}(1)-\mathrm{Cl}(1)$ & $86.859(36)$ \\
$\mathrm{Cl}(1)-\mathrm{Ru}(1)-\mathrm{N}(3)$ & $84.623(77)$ \\
\hline
\end{tabular}

从晶体堆积图(图 2)上可见, 配合物的分子之间为 数众多的氢键作用 $[\mathrm{N}(1)-\mathrm{H}(2 \mathrm{a}) \cdots \mathrm{Cl}(2)(2.360 \AA)$, $\mathrm{C}(4)-\mathrm{H} \cdots \mathrm{Cl}(2)(2.848 \AA), \mathrm{C}(9)-\mathrm{H} \cdots \mathrm{Cl}(2)(2.819 \AA)$ 和 $\mathrm{C}(6)-\mathrm{H} \cdots \mathrm{Cl}(1 \mathrm{~A})(2.722 \AA)]$ 使配合物晶体具有较高的 稳定性. 配合物的其他晶体数据见 CCDC 993970(从 deposit@ccdc.cam.ac.uk 获得).

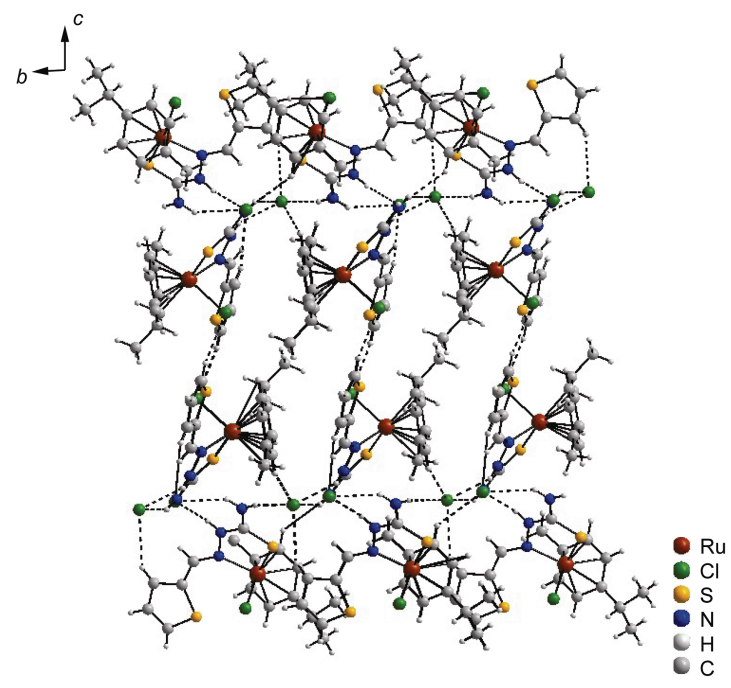

图 2 化合物 1 的分子堆积图

Figure 2 Crystal packing pictures of 1
我们通过琼脂糖凝胶电泳法监测超螺旋环状 DNA (Form I) 转化为开链环状 DNA (Form II) 和线型 DNA (Form III)的程度, 研究 2-甲酰噻吩 TSC 化合物 L1 和 L2 及其芳基钓配合物 $\mathbf{1}$ 和 $\mathbf{2}$ 在生理条件下对 pBR322 质粒 DNA 的影响. 琼脂糖凝胶电泳结果见图 3. 与作为 空白对照的 pBR322 质粒 DNA 相比(lane 1), lane 2 (1) 只 出现较浅的 Form I 印迹, 观察不到 Form II 和 Form III; 而 lane 3 (2)则完全观察不到 Form I, Form II 和 Form III 的印迹, 这意味着配合物 $\mathbf{1}$ 和 $\mathbf{2}$ 对质粒 DNA 有较为彻 底的裂解作用. ${ }^{[14]}$ 值得注意的是, L1 和 L2 (lane 4, lane 5)的琼脂凝胶电泳结果与空白对照一致, 说明 2-甲酰噻 吩 TSC 化合物单体并不改变 DNA 的三级结构. 琼脂糖 凝胶电泳实验表明, 2-甲酰噻吩 TSC 化合物作为单体及 其形成芳基钓配合物后与 pBR322 质粒 DNA 的作用机 理是不同的.

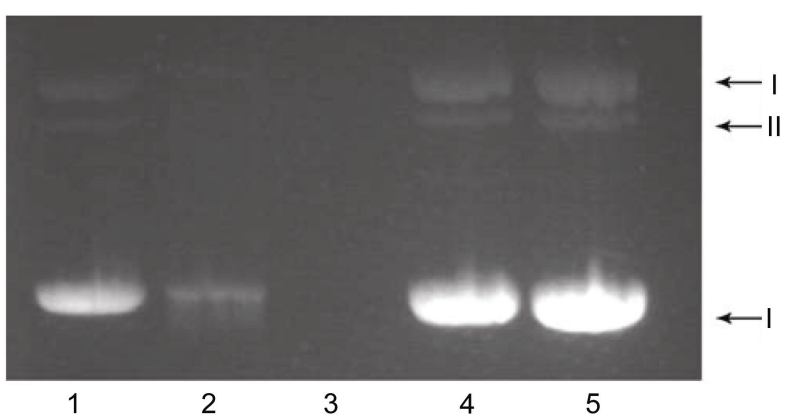

图 32 2-甲酰噻吩 TSC 化合物单体及其芳基钉配合物 1, 2 对 pBR322 质粒 DNA 的电泳迁移图

Figure 3 Electrophoretic mobility pattern of pBR322 plasmid DNA incubated with 2-formylthiophene TSC monomers and their ruthenium(II) arene complexes $\mathbf{1}, \mathbf{2}$

lane 1: pBR322; lane 2: 1; lane 3: 2; lane 4: L1; lane 5: L2

2-甲酰噻吩 TSC 化合物及其芳基钓配合物的体外 抗肿瘤活性见表 3. 结果显示, 作为 TSC 单体的 L1 和 L2 对人肝癌细胞株(BEL 7404)和人鼻咽癌细胞株 (CNE-1)均未表现出抗肿瘤活性, 只对人胃癌细胞株 (SGC7901) 表现出一定抗肿瘤活性，其 $\mathrm{IC}_{50}$ 值分别为 60.3 和 $75.7 \mu \mathrm{mol} \cdot \mathrm{L}^{-1}$. 与芳基钓配位形成相应的配合 物 $\mathbf{1}$ 和 $\mathbf{2}$ 以后，其对上述几种肿瘤细胞生长的抑制活性 发生了变化. 1 对人胃癌细胞株(SGC7901)的抑制活性比 其相应的 TSC 配体单体稍差, $\mathrm{IC}_{50}$ 值为 $73.4 \mu \mathrm{mol} \cdot \mathrm{L}^{-1}$; 对人肝癌细胞株(BEL7404)也没有活性. 值得注意的是, 1 对人鼻咽癌细胞株(CNE-1)的抑制活性有较为明显的 提高, 其 $\mathrm{IC}_{50}$ 值为 $27.8 \mu \mathrm{mol} \cdot \mathrm{L}^{-1}$, 与作为对照的顺铂的 细胞抑制相当. 配合物 2 则恰恰相反, 其对 SGC7901, BEL7404 和 CNE-1 三种肿瘤细胞均无抑制活性. 由此 可见，这两个2-甲酰噻吩 TSC 化合物的抗肿瘤活性有选 择地对某些肿瘤细胞显示抑制活性. 这可能是因为 TSC 
分子本征的疏水作用使其不易透过细胞膜，抑制了分子 的活性 ${ }^{[15]}$. 形成芳基钓配合物后，化合物分子性质发生 改变, 其对细胞膜的透过作用、对肿瘤细胞的作用机理 发生改变, 导致对肿瘤细胞的抑制活性有较大程度的变 化. 从电泳结果来看, 与配合物 1 一样, 配合物 2 对 DNA 裂解十分完全, 但是对上述三种肿瘤细胞的抑制 作用却与 1 有明显的差异, 这可能是由于分子结构的改 变, 使得分子的疏水性、位阻等本征性质发生改变, 引起 其在细胞膜的透过性及与 DNA 的结合能力发生变化所 导致. 因此, 2-甲酰噻吩 TSC 化合物及其芳基钓配合物的 构效关系还有待在综合考虑取代基团的种类、数目和疏 水作用等多方面因素的基础上，设计、合成更多的 TSC 化合物及其芳基钉配合物, 再进行更深入的研究.

表 3 TSC 单体与相应芳基钉配合物的体外抗肿瘤活性 $\left[\mathrm{IC}_{50} /\left(\mu \mathrm{mol} \cdot \mathrm{L}^{-1}\right)\right]$

Table 3 In vitro inhibition activities of tumor for TSCs and corresponding ruthenium arene complexes $\left[\mathrm{IC}_{50} /\left(\mu \mathrm{mol} \cdot \mathrm{L}^{-1}\right)\right]$

\begin{tabular}{lccc}
\hline Compd. & SGC7901 & BEL7404 & CNE-1 \\
\hline L1 & 60.3 & $>100$ & $>100$ \\
L2 & 75.7 & $>100$ & $>100$ \\
$\mathbf{1}$ & 73.4 & $>100$ & 27.8 \\
$\mathbf{2}$ & $>100$ & $>100$ & $>100$ \\
Cisplatin & 6.7 & 23.2 & 30.1 \\
\hline
\end{tabular}

\section{2 结论}

合成了两个 2-甲酰噻吩 TSC 化合物 L1, L2 及其芳基 钉配合物 1, 2. 利用单晶衍射法研究了 1 的分子结构. 凝 胶电泳实验表明, $\mathbf{L 1}$ 和 $\mathbf{L 2}$ 作为单体对 $\mathrm{pBR} 322$ 质粒 DNA 的作用与其形成芳基钉配合物 1 和 $\mathbf{2}$ 后的作用机理 有明显差异. 对这些化合物的体外抗肿瘤活性进行了对 比研究, 其中配合物 $\mathbf{1}$ 表现出较好的抗肿瘤活性. 该研究 结果将为我们进一步改变分子结构设计与合成活性更高 的有机金属芳基钉化合物分子提供理论依据.

\section{3 实验部分}

\section{1 仪器与试剂}

$\mathrm{RuCl}_{3} \cdot x \mathrm{H}_{2} \mathrm{O}$ 和氨基硫脲均从百灵威公司购买, 其 它试剂均为市售分析纯.

Bruker AV-300 核磁共振仪(300 MHz); Waters UPLC XEVO G2 TOF 质谱仪; Elementar Vario EL Cube 元素分析仪; Agilent 8453 紫外光谱仪. [( $\eta^{6}-p$-cymene)$\left.\mathrm{RuCl}_{2}\right]_{2}$ 按文献[12]方法合成.

\subsection{TSC 配体 L1 和 L2 的合成}

TSC 配体 L1 和 L2 均按文献[12]方法合成. $100 \mathrm{~mL}$ 三口烧瓶中加入相应的氨基硫艮 (1 mmol), 2-甲酰噻吩 (1 mmol), 甲醇 $(5 \mathrm{~mL})$, 滴入 $1 \sim 2$ 滴醋酸, 加热回流 $3 \mathrm{~h}$,
出现白色沉淀. 冷却后转移至单口烧瓶, 减压蒸馏除去 大部分溶剂(剩余 $1 \mathrm{~mL}$ ), 冷却重结晶得到产物. 表征数 据同文献[13].

\section{3 氯化 [-氯-一-2-甲酰噻吩缩氨基硫服-甲基异丙基 苯合钉(II)] (1)的合成}

$\left[\mathrm{Ru}\left(\eta^{6} \text {-p-cymene }\right) \mathrm{Cl}_{2}\right]_{2}(0.05 \mathrm{mmol}, 31.5 \mathrm{mg})$ 与 2-甲 酰噻吩醛缩氨基硫腿( $(\mathbf{1}, 0.1 \mathrm{mmol}, 47 \mathrm{mg}$ )溶于 $8 \mathrm{~mL}$ 二 氯甲烷中, 在 $45{ }^{\circ} \mathrm{C}$ 下回流 $6 \mathrm{~h}$, 冷却后用石油醚重结晶, 得 $35.3 \mathrm{mg}$ 红棕色的晶体, 产率 $72 \%$. m.p. 190 191 ${ }^{\circ} \mathrm{C}$; UV-vis $(\mathrm{MeOH}) \lambda_{\max }: 215,346 \mathrm{~nm} ;{ }^{1} \mathrm{H}$ NMR $(300 \mathrm{MMz}$, $\left.\mathrm{CDCl}_{3}\right) \delta: 9.27(\mathrm{~s}, 1 \mathrm{H}, \mathrm{CH}=\mathrm{N}), 8.35(\mathrm{~d}, J=3.3 \mathrm{~Hz}, 1 \mathrm{H}$, thiophene-H), $7.27 \sim 7.23(\mathrm{~m}, 1 \mathrm{H}$, thiophene-H), $7.79(\mathrm{~d}$, $J=5.0 \mathrm{~Hz}, 1 \mathrm{H}$, thiophene-H), $5.59(\mathrm{~d}, J=5.7 \mathrm{~Hz}, 1 \mathrm{H}$, p-cymene), 5.50 (d, $J=5.8 \mathrm{~Hz}, 1 \mathrm{H}$, phenyl-H), 5.36 (d, $J=5.8 \mathrm{~Hz}, 1 \mathrm{H}$, phenyl-H), 5.30 (d, $J=5.7 \mathrm{~Hz}, 2 \mathrm{H}$, phenyl-H), $2.82 \sim 2.70\left[\mathrm{~m}, 1 \mathrm{H}, \mathrm{CH}\left(\mathrm{CH}_{3}\right)_{2}\right], 2.17(\mathrm{~s}, 3 \mathrm{H}$, $\left.\mathrm{CH}_{3}\right), 1.23$ [d, $\left.J=6.8 \mathrm{~Hz}, 3 \mathrm{H}, \mathrm{CH}\left(\mathrm{CH}_{3}\right)_{2}\right], 1.18$ [d, $J=6.8$ $\left.\mathrm{Hz}, 3 \mathrm{H}, \mathrm{CH}\left(\mathrm{CH}_{3}\right)_{2}\right]$; MS (ESI) $m / z(\%): 420.1\left([\mathrm{M}-\mathrm{HCl}]^{+}\right.$, 100). Anal. calcd for $\mathrm{C}_{16} \mathrm{H}_{21} \mathrm{Cl}_{2} \mathrm{~N}_{3} \mathrm{RuS}_{2} \cdot \mathrm{H}_{2} \mathrm{O}: \mathrm{C} 37.72, \mathrm{H}$ 4.55, N 8.25; found C 37.62, H 4.47, N 8.47.

\section{4 氯化 [一氯- - - 2-甲酰噻吩缩- $N^{4}$-甲基-氨基硫服 - 甲基异丙基苯合钉(II)] (2)的合成}

$\left[\mathrm{Ru}\left(\eta^{6}-p \text {-cymene }\right) \mathrm{Cl}_{2}\right]_{2}(0.05 \mathrm{mmol}, 31.5 \mathrm{mg})$ 与 2-甲 酰塞吩醛缩- $N^{4}$-甲基-氨基硫脲 $(\mathbf{L 1}, 0.1 \mathrm{mmol}, 47 \mathrm{mg}$ )溶 于 $10 \mathrm{~mL}$ 二氯甲烷中, 在 $55{ }^{\circ} \mathrm{C}$ 下回流 $12 \mathrm{~h}$, 冷却后用 石油醚重结晶, 得 $28.3 \mathrm{mg}$ 红棕色的晶体, 产率 $56 \%$. m.p. $201 \sim 202{ }^{\circ} \mathrm{C}$; UV-vis (MeOH) $\lambda_{\max }: 215,359 \mathrm{~nm} ;{ }^{1} \mathrm{H}$ NMR (300 MMz, $\left.\mathrm{CDCl}_{3}\right) \delta$ : 14.07 (br, 1H, NH), 9.00 (s, $1 \mathrm{H}, \mathrm{CH}=\mathrm{N}), 8.68\left(\mathrm{br}, 1 \mathrm{H}, \mathrm{NHCH}_{3}\right), 8.33(\mathrm{~d}, J=3.3 \mathrm{~Hz}$, $1 \mathrm{H}$, thiophene-H), $7.77(\mathrm{~d}, J=4.1 \mathrm{~Hz}, 1 \mathrm{H}$, thiophene-H), 7.29 (dd, $J=10.6,4.7 \mathrm{~Hz}, 1 \mathrm{H}$, thiophene-H), 5.56 (dd, $J=$ 5.6, $12.8 \mathrm{~Hz}, 1 \mathrm{H}$, phenyl-H), 5.29 (d, $J=5.7 \mathrm{~Hz}, 1 \mathrm{H}$, phenyl-H), 5.13 (t, $J=4.7 \mathrm{~Hz}, 2 \mathrm{H}$, phenyl-H), 3.15 (d, $J=$ $\left.3.9 \mathrm{~Hz}, 3 \mathrm{H}, \mathrm{NHCH}_{3}\right), 2.82 \sim 2.71\left[\mathrm{~m}, 1 \mathrm{H}, \mathrm{CH}\left(\mathrm{CH}_{3}\right)_{2}\right], 2.16$ (s, $\left.3 \mathrm{H}, \mathrm{CH}_{3}\right), 1.24$ [d, $\left.J=6.8 \mathrm{~Hz}, 3 \mathrm{H}, \mathrm{CH}\left(\mathrm{CH}_{3}\right)_{2}\right], 1.19$ [d, $J=6.8 \mathrm{~Hz}, 3 \mathrm{H}, \mathrm{CH}\left(\mathrm{CH}_{3}\right)_{2}$ ]; $\mathrm{MS}$ (ESI) $m / z(\%): 434.0$ $\left([\mathrm{M}-\mathrm{HCl}]^{+}, 100\right)$. Anal. calcd for $\mathrm{C}_{17} \mathrm{H}_{23} \mathrm{Cl}_{2} \mathrm{~N}_{3} \mathrm{RuS}_{2}$ : C 40.39, H 4.59, N 8.31; found C 40.00, H 4.53, N 8.30.

\section{5 单晶测试}

单晶测试在 Oxford Diffraction Gemini E system 上 进行, 用单色化 $\mathrm{CuK \alpha}(\lambda=1.5418 \AA)$, 测试温度为 293 K. 晶体结构用直接法和 Fourier 法解出, 各向异性热参 数进行全矩阵最小二乘法修正, 全部非氢原子的坐标、 各向异性温度因子和氢原子的坐标及各向同性温度因 
子修正至收玫, 氢原子从差 Fourier 图上定出, 所有计算 均在 PC 计算机上用 SHELXTL-97 程序进行 ${ }^{[16]}$.

\subsection{DNA 裂解实验}

化合物对 $\mathrm{pBR} 322$ 质粒 DNA 的作用条件为: 化合物 浓度为 $0.8 \mathrm{mg} / \mathrm{mL}, \mathrm{pBR} 322$ 质粒 DNA 浓度为 $0.5 \mu \mathrm{g} / \mu \mathrm{L}$, 在 EP 管中依次加入相应的化合物 $(2 \mu \mathrm{L}) 、 \mathrm{pBR} 322$ 质粒 DNA $(1.5 \mu \mathrm{L})$, 混匀后放入恒温水槽内, 控制温度 $(37 \pm 0.1){ }^{\circ} \mathrm{C}$, 反应 $45 \mathrm{~min}$. 取 pBR322 质粒 DNA 1.5 $\mu \mathrm{L}$, 化合物与 $\mathrm{pBR} 322$ 质粒 DNA 混合液各 $3.5 \mu \mathrm{L}$, 与一 定量的加样缓冲液混匀后, 用微量移液器小心加入凝胶 样品孔中, 盖上电泳槽, 于 $60 \mathrm{~V}$ 恒电压下电泳 $2.5 \mathrm{~h}$. 在 紫外灯下观察, 用 CCD 成像系统照相.

\section{7 体外抗肿瘤活性}

通过 MTT 法测定化合物对三种肿瘤细胞株的细胞 毒活性来评价其体外抗肿瘤活性, 细胞株分别为人胃癌 细胞株(SGC7901)、人肝癌细胞株(BEL7404)和人鼻咽癌 细胞株(CNE-1). 将肿瘤细胞分别在盛有 1640 培养液 [内含 $10 \%$ (体积分数)胎牛血清]的培养瓶中培养, 培养 条件为 $37{ }^{\circ} \mathrm{C}, 5 \% \mathrm{CO}_{2}$, 取对数期生长的癌细胞经 $0.25 \%$ 的胰蛋白酶液消化后，用 1640 培养液(内含 $10 \%$ 胎牛血清)配成细胞悬液, 加入 96 孔板, 每孔 $200 \mu \mathrm{L}$, $1 \times 10^{4} \sim 3 \times 10^{4}$ 个细 胞/孔, 置培养箱内培养 $24 \mathrm{~h}$; 采 用 MTT 法, 每孔加入 $1 \mu \mathrm{L}$ 浓度不同的药物, 药物终浓 度分别为 $10,20,30,50,60,80 \mu \mathrm{mol} \cdot \mathrm{L}^{-1}$, 培养箱中培养 $24 \mathrm{~h}$. 每孔加入 $20 \mu \mathrm{L}(5 \mathrm{mg} / \mathrm{mL}) \mathrm{MTT}$, 再置培养箱中培 养 $4 \mathrm{~h}$, 吸掉上清液, 每孔加入 $100 \mu \mathrm{L}$ 的 DMSO 置摇床 上低速震荡, $10 \mathrm{~min}$ 后在酶标仪上用 $492 \mathrm{~nm}$ 波长测 $\mathrm{OD}$ 值. 每种药物重复 3 次实验, 每次做 3 个复孔.

\section{References}

[1] (a) Beraldo, H.; Gambino, D. Mini-Rev. Med. Chem. 2004, 4, 31. (b) Yu, Y.; Kalinowski, D. S.; Kovacevic, Z.; Siafakas, A. R.; Jansson, P. J.; Stefani, C.; Lovejoy, D. B.; Sharpe, P. C.; Bernhardt, P. V.; Richardson, D. R. J. Med. Chem. 2009, 52, 5271.

(c) Kalinowski, D. S.; Quach, P.; Richardson, D. R. Future Med. Chem. 2009, 1, 1143.

[2] (a) Ren, S.; Wang, R.; Komatsu, K.; Bonaz-Krause, P.; Zyrianov, Y.; McKenna, C. E.; Csipke, C.; Tokes, Z. A.; Lien, E. J. J. Med. Chem. 2002, 45, 410 .

(b) Huang, H.; Chen, Q.; Ku, X.; Meng, L.; Lin, L.; Wang, X.; Zhu, C.; Wang, Y.; Chen, Z.; Li, M.; Jiang, H.; Chen, K.; Ding, J.; Liu, H. J. Med. Chem. 2010, 53, 3048.

[3] (a) Adsule, S.; Barve, V.; Chen, D.; Ahmed, F.; Dou, Q. P.; Padhye, S.; Sarkar, F. H. J. Med. Chem. 2006, 49, 7242.

(b) Easmon, J.; Purstinger, G.; Heinisch, G.; Roth, T.; Fiebig, H. H.; Holzer, W.; Jager, W.; Jenny, M.; Hofmann, J. J. Med. Chem. 2001, 44, 2164.

[4] (a) Beckford, F. A.; Leblanc, G.; Thessing, J.; Shaloski Jr., M.; Frost, B. J.; Li, L.; Seeram, N. P. Inorg. Chem. Commun. 2009, 12, 1094. (b) Zeglis, B. M.; Divilov, V.; Lewis, J. S. J. Med. Chem. 2011, 54, 2391.

[5] (a) Hartinger, C. G.; Zorbas-Selfried, S.; Jakupee, M. A.; Kynast, B.; Zorbas, H.; Keppler, B. K. J. Inorg. Biochem. 2006, 100, 891.

(b) Yan, Y. K.; Melchart, M.; Habtemariam, A.; Sadler, P. J. Chem. Commun. 2005, 4764.

(c) Hotze, A. C. G.; Kariuki, B. M.; Hannon, M. J. Angew. Chem. 2006, 118, 4957 .

(d) Vajpayee, V.; Yang, Y. J.; Kang, S. C.; Kim, H.; Kim, I. S.; Wang, M.; Stang, P. J.; Chi, K.-W. Chem. Commun. 2011, 47, 5184.

[6] (a) Rademaker-Lakhai, J. M.; Van Den Bongard, D.; Pluim, D.; Beijnen, J. H.; Schellens, J. H. M. Clin. Cancer Res. 2004, 10, 3717.

(b) Hartinger, C. G.; Jakupec, M. A.; Zorbas-Seifried, S.; Groessl, M.; Egger, A.; Berger, W.; Zorbas, H.; Dyson, P. J.; Keppler, B. K. Chem. Biodiversity 2008, 5, 2140.

(c) Suess-Fink, G. Dalton Trans. 2010, 39, 1673

[7] (a) Clarke, M. J. Coord. Chem. Rev. 2003, 36, 209.

(b) Wang, F.; Xu, J.; Habtemariam, A.; Bella, J.; Sadler, P. J. J. Am. Chem. Soc. 2005, 127, 17734.

(c) Liu, M.; Lim, Z. J.; Gwee, Y. Y.; Levina, A.; Lay, P. A. Angew. Chem., Int. Ed. 2010, 49, 1661.

(d) Hartinger, C. G.; Metzler-Nolte, N.; Dyson, P. J. Organometallics 2012, 31, 5677.

[8] (a) Peacock, A. F. A.; Melchart, M.; Deeth, R. J.; Habtemariam, A.; Parsons, S.; Sadler, P. J. Chem. Eur. J. 2007, 13, 2601.

(b) Fernandez, R.; Melchart, M.; Habtemariam, A.; Parsons, S.; Sadler, P. J. Chem. Eur. J. 2004, 10, 5173.

(c) Bugarcic, T.; Habtemariam, A.; Stepankova, J.; Heringova, P.; Kasparkova, J.; Deeth, R. J.; Johnstone, R. D. L.; Prescimone, A.; Parkin, A.; Parsons, S.; Brabec, V.; Sadler, P. J. Inorg. Chem. 2008 47, 11470 .

(d) Kljun, J.; Bytzek, A. K.; Kandioller, W.; Bartel, C.; Jakupec, M. A.; Hartinger, C. G.; Keppler, B. K.; Turel, I. Organometallics 2011, 30, 2506.

[9] (a) Gossens, C.; Tavernelli, I.; Rothlisberger, U. J. Am. Chem. Soc. 2008, 130, 10921.

(b) Chen, H.; Parkinson, J. A.; Parsons, S.; Coxall, R. A.; Gould, R. O.; Sadler, P. J. J. Am. Chem. Soc. 2002, 125, 3064.

[10] (a) Liu, H. K.; Berners-Price, S. J.; Wang, F. Y.; Parkinson, J. A.; Xu, J. J.; Bella, J.; Sadler, P. J. Angew. Chem., Int. Ed. 2006, 45, 8153.

(b) Liu, H.-K.; Parkinson, J. A.; Bella, J.; Wang, F.; Sadler, P. J. Chem. Sci. 2010, 1, 258.

[11] (a) Dougan, S. J.; Habtemariam, A.; McHale, S. E.; Parsons, S.; Sadler, P. J. Proc. Natl. Acad. Sci. U. S. A. 2008, 105, 11628.

(b) Giannini, F.; Suss-Fink, G.; Furrer, J. Inorg. Chem. 2011, 50, 10552 .

[12] (a) Beckford, F.; Dourth, D.; Shaloski Jr., M.; Didion, J.; Thessing, J.; Woods, J.; Crowell, V.; Gerasimchuk, N.; Gonzalez-Sarrías, A.; Seeram, N. P. J. Inorg. Biochem. 2011, 105, 1019.

(b) Stringer, T.; Therrien, B.; Hendricks, D. T.; Guzgay, H.; Smith, G. S. Inorg. Chem. Commun. 2011, 14, 956.

(c) Su, W.; Zhou, Q.; Huang, Y.; Huang, Q.; Huo, L.; Xiao, Q.; Huang, S.; Huang, C.; Chen, R.; Qian, Q.; Liu, L.; Li, P. Appl. Organomet. Chem. 2013, DOI: 10.1002/aoc.2977.

[13] (a) Mathew, M.; Palenik, G. J. Acta Crystallogr. 1971, B27, 59. (b) Li, M.-X.; Zhang, D.; Zhang, L.-Z.; Niu, J.-Y. Inorg. Chem. Commun. 2010, 13, 1268.

[14] Xiang, Q.-X.; Zhang, L.-Q.; Yu, X.-Q.; Xie, R.-G. Chin. J. Org. Chem. 2009, 29, 580 (in Chinese). 
(向清祥, 张丽群, 余孝其, 谢如刚, 有机化学, 2009, 29, 580.)

[15] Alomar, K.; Landreau, A.; Kempf, M.; Khan, M. A.; Allain, M.; Bouet, G. J. Inorg. Biochem. 2010, 104, 397.
[16] Sheldrick, G. M. SHELX97: Program System for Crystal Structure Determination, University of Göttingen, Göttingen, Germany, 1997.

(Zhao, C.) 